\title{
An Improved Equivalent Fixture Error Model for Machining Process
}

\author{
Fuyong Yang ${ }^{1,2}$, Sun $\mathrm{Jin}^{1,2, *}$, Zhimin $\mathrm{Li}^{1,2}$, Siyi Ding ${ }^{1,2}$, Jia Lin $^{1,2}$, Shun Liu $^{1,2}$ \\ ${ }^{1}$ State Key Laboratory of Mechanical System and Vibration, Shanghai Jiao Tong University, Shanghai, 200240, P.R. China \\ ${ }^{2}$ Shanghai Key Laboratory of Digital Manufacture for Thin-walled Structures, Shanghai Jiao Tong University, Shanghai, 200240, P.R. \\ China
}

\begin{abstract}
Although the equivalent fixture error approach used in variation propagation can directly model the process physics regarding how datum-induced, fixture-induced and machine tool-induced errors generate the same error pattern on part features, the developed equivalent fixture error compensation technique did not consider machining-induced variations. Machining-induced variations are often caused by geometric-thermal effects, cutting force-induced variations, and/or cutting-tool wear, etc. Such machininginduced variations are an important factor that influences the part quality. Without considering machininginduced variations, the application of EFE model for error compensation will be limited. In order to overcome this limitation, this paper extends current equivalent fixture error to include machining-induced variations. This paper shows the benefits of the extended model through a case study.
\end{abstract}

\section{Introduction}

For a multistage machining process (MMPs), the part variation at certain operation is often due to two components: 1) the variation brought by current machine stage, such as fixture error and machine tool path error; and 2) the variation brought by datum feature error generated and propagated from previous stages [1]. The above errors account for the majority of machining errors in the machining process. Due to the existence of these main error sources, the features of the workpiece may deviate from their designed values to their actual values.

SPC is a very useful tool for monitoring process variation and reducing product variation [2-4]. Tsung et al. [5] gave a thorough review about the application of SPC techniques in MMPs, including MMPs and multistage service operations. However, SPC could not be directly used to model the relationship between the error sources and feature variations. It also cannot handle variation propagation problem in MMPs.

$\mathrm{SoV}$ is another useful technology for reducing product variation and can overcome the limitation of SPC. This technology can directly model how the variation in MMPs caused by datum, fixture, and machine path tool propagate and accumulate. The SoV methodology for MMPs can be described by employing the state space concept which has been extensively used in system or automatic control theories. The introduction of the state space modeling structure can successfully describe the relationship between the error sources and feature variations. A huge of literature can be found on SoV modeling. Jin et al. [6] introduced state space model to describe the dimensional variation accumulation and propagation for multistage body assembly processes. To obtain the SoV models for MMPs, lots of efforts have been done [1, 7-12]. Huang et al. [7] proposed an implicit non-linear SoV model to predict the variation accumulation and its propagation for MMPs. Djurdjanovic et al. [8] used Taylor series expansion to linearize Huang et al.'s non-linear SoV model. But the linearized model is yet implicit. Using differential motion vectors (DMVs), Zhou et al. [1] developed an explicit linear SoV model. Abellan-Nebot et al. [9] demonstrated that the absence of machining-induced variations in state space modeling of variation propagation for MMPs could be an important factor that influences the accurate in variation prediction. Using DMVs, Abellan-Nebot et al. [10] introduced such machining-induced variations including geometricthermal effects, cutting force-induced variations, and/or cutting-tool wear, etc into the SoV model. Several review papers have introduced $\mathrm{SoV}$ modeling methods briefly [11-14].

To reduce this variation, Wang et al. [15, 16] proposed equivalent fixture error (EFE) concept. With this concept, datum error and machine tool error can be transformed to equivalent fixture locator errors at each operation and be compensated. However, only the datum errors and geometric errors of machine tool have been explicitly transformed to equivalent fixture locator variations at each operation in the reported works [15, 16], leaving the machining-induced variations unaddressed. Therefore, without considering machininginduced variations, the application of current EFE model for error compensation will be limited.

Corresponding author: jinsun@sjtu.edu.cn 
In order to overcome this limitation of current EFE compensation, this paper extends current EFE model to include machining-induced variations. By this extended EFE model, the machining-induced variations can be cancelled out by their corresponding EFE. Then, the machining quality can correspondingly improve. The limitation of current EFE model and the benefits of extending the EFE model through a case study will also be discussed in this paper.

\section{Related works}

\subsection{EFE model}

The concept of EFE introduced by $[15,16]$ for machining process can directly model the process physics regarding how datum error, and machine tool path error, and fixture error generate the same pattern on the machined features. Through the EFE model, datum error and machine tool path error can be transformed to equivalent fixture locator errors at each operation.

In Fig. 1(a), $\mathbf{X}_{1}^{0}, \mathbf{X}_{2}^{0}, \mathbf{X}_{3}^{0}$ and $\mathbf{X}_{4}^{0}$ (the solid line block with surfaces) are the nominal features in PCS. $\mathbf{X}_{1}^{0}$ and $\mathbf{X}_{2}^{0}$ are the designed primary and secondary datum features, respectively. When the datum features and the fixture are all at their designed positions, the part shown in Fig. 1(a) will be in its nominal setup position. However, when the datum feature $\mathbf{X}_{1}$ in Fig. 1(b) deviates from its nominal, the block will be transformed to its actual position. As shown in Fig. 1(c), the datum error can be equivalent to the fixture error $\Delta d_{1}$ and $\Delta d_{2}$.

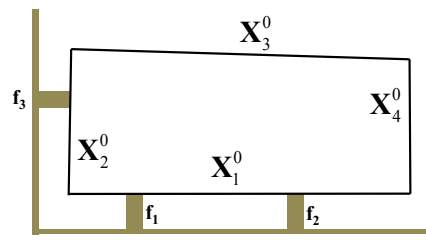

(a)

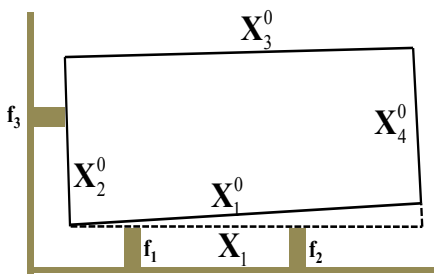

(b)

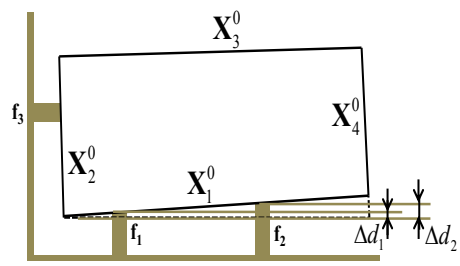

(c)

Figure. 1. EFE due to datum error: (a) nominal setup, (b) setup error caused by datum error, (c) setup error caused by the EFE of the datum error
Similarly, the machine tool path error shown in Fig. 2(a) can also be equivalent to its corresponding equivalent fixture locator errors shown in Fig. 2(b).

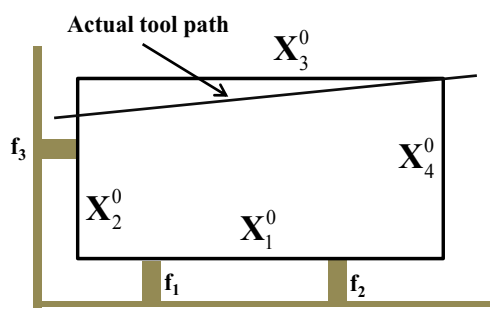

(a)

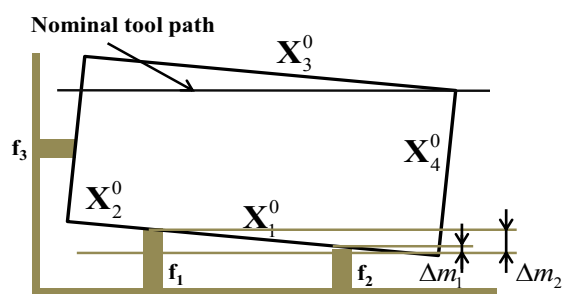

(b)

Figure. 2. EFE due to machine tool path error: (a) machining error caused by machine tool path error, (b) machining error caused by the EFE of the machine tool path error

The EFE model for datum error, fixture error and machine tool path error can be described as follows.

$\mathbf{x}_{i}(k)=\mathbf{B}_{i}(k)\left[\begin{array}{ll}\Delta \mathbf{d}^{\mathrm{T}}(k) & 0\end{array}\right]^{\mathrm{T}}+\mathbf{B}_{i}(k) \mathbf{u}(k)+\xi_{i}(k)$

where $\quad \mathbf{B}_{i}(k)=\left[\begin{array}{cc}-\mathbf{A}_{i d}(k) \mathbf{J}^{-1}(k) \boldsymbol{\Phi}(k) \mathbf{E} & \mathbf{0}_{6 \times 1} \\ \mathbf{0}_{1 \times 6} & \gamma_{i}^{0}(k)\end{array}\right]$ is the coefficient matrix linking the errors with the machined feature deviation. $\operatorname{rank}\left(-\mathbf{A}_{i d}(k) \mathbf{J}^{-1}(k) \boldsymbol{\Phi}(k) \mathbf{E}\right) \leq 5$ and $\mathbf{u}(k)=\left[(\Delta \mathbf{m}(k)+\Delta \mathbf{f}(k))^{\mathrm{T}}, \gamma_{m}(k)-1\right]^{\mathrm{T}}$. The expression of $\Delta \mathbf{d}(k)$ and $\Delta \mathbf{m}(k)$ are as follows.

$$
\Delta \mathbf{m}(k)=\mathbf{K} \delta \mathbf{q}_{m}(k)
$$

$\left[\begin{array}{c}\Delta \mathbf{d}(k) \\ 1\end{array}\right]=\left[\begin{array}{cc}\boldsymbol{\Psi} & \mathbf{0} \\ \mathbf{0} & \mathbf{1}\end{array}\right]_{7 \times 22} \mathbf{H}_{22 \times 22}\left[\begin{array}{cc}\mathbf{G} & \mathbf{0} \\ \mathbf{0} & \mathbf{1}\end{array}\right]_{22 \times 22}\left[\begin{array}{c}\mathbf{x}_{1}(k-1) \\ \mathbf{x}_{2}(k-1) \\ \mathbf{x}_{3}(k-1) \\ 1\end{array}\right]_{22 \times 1}$

where $\delta \mathbf{q}_{m}(k)$ is machine path tool error, $\mathbf{x}_{1}(k-1)$, $\mathbf{x}_{2}(k-1)$ and $\mathbf{x}_{3}(k-1)$ are the primary datum error, secondary datum error and tertiary datum error, respectively. The detailed expression of matrix $\mathbf{K}, \boldsymbol{\Psi}$, $\mathbf{H}$ and $\mathbf{G}$ can be found in [15].

\subsection{Compensation strategy}

To compensate the error sources, corresponding $\Delta \mathbf{d}$, $\Delta \mathbf{f}(k)$ and $\Delta \mathbf{m}$ should be calculated by EFE model. Denote the total EFE as $\mathbf{t}(k)=[\Delta \mathbf{d}(k)+\Delta \mathbf{f}(k)+\Delta \mathbf{m}(k)]$. In order to cancel out $\mathbf{t}(k)$, the amount of adjustment $\mathbf{c}(k)=-[\Delta \mathbf{d}(k)+\Delta \mathbf{f}(k)+\Delta \mathbf{m}(k)]$ should be applied to the corresponding fixture locators. The detailed compensation process by EFE model is shown in Fig. 3. 
For example, to compensate the datum error shown in in Fig. 1(b), $\Delta \mathbf{d}$ should be calculated. When the amount of adjustment $-\Delta \mathbf{d}$ shown in Fig. 4 is applied to the corresponding fixture locators, $\Delta \mathbf{d}$ can be cancelled out. Then, the datum error can be compensated by this strategy. The same procedure can be performed to compensate the machine tool path error $\mathbf{u}_{m}$.

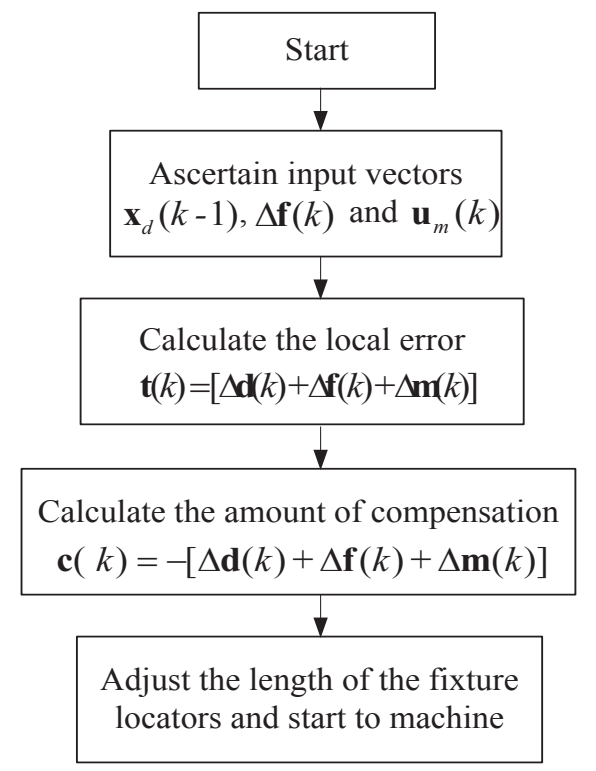

Figure. 3. The compensation process by EFE

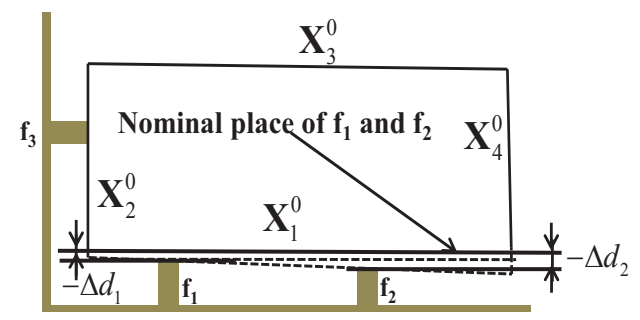

Figure. 4. The adjustment to compensate datum error

\section{Improved Equivalent Fixture Error Model}

In order to overcome the limitation of current EFE model, this paper extends current EFE model to include machining-induced variations. Abellan-Nebot et al. [10] presented a generic framework for machining-induced variation representation based on DMVs. The final machine tool path error caused by machining-induced variations is mathematically modeled as

$$
\delta \mathbf{q}_{m}(k)=\mathbf{T}_{q i(k)}^{C_{k}}\left(\mathbf{T}_{C_{k}}^{S_{k}}\left(\mathbf{T}_{S_{k}}^{A_{k}^{n}} \mathbf{x}_{A_{k}^{o}}^{o} F_{k}+\mathbf{x}_{S_{k}}^{A_{k}^{n}}\right)+\mathbf{x}_{C_{k}}^{S_{k}}\right)+\mathbf{x}_{q i(k)}^{C_{k}}
$$

where $\mathbf{x}_{A_{k}^{n}}^{o} F_{k}$ is the geometric-thermal variation of machine-tool axes; $\mathbf{x}_{S_{k}}^{A_{k}^{n}}$ represents the spindle-thermal variation; $\mathbf{x}_{C_{k}}^{S_{k}}$ refers to the cutting force-induced variation and $\mathbf{x}_{q i}^{C_{k}}$ is the cutting-tool wear induced variations; $\mathbf{T}_{q i(k)}^{C_{k}}, \mathbf{T}_{C_{k}}^{S_{k}}$ and $\mathbf{T}_{S_{k}}^{A_{k}^{n}}$ are the matrices to describe the transition of differential motion vectors can be found in [10].

It can be seen from Eq. 4 that when $\mathbf{x}_{A_{k}^{n}}^{{ }^{\circ} F_{k}}=\mathbf{0}$, $\mathbf{x}_{S_{k}}^{A_{k}^{n}}=\mathbf{0}$ and $\mathbf{x}_{C_{k}}^{S_{k}}=\mathbf{0}$, the machining tool path error due to cutting-tool wear can be written as

$$
\delta \mathbf{q}_{m}^{q i(k)}(k)=\mathbf{x}_{q i(k)}^{C_{k}}
$$

Similarly, the machine tool path error due to cutting force-induced variation, spindle-thermal variation and geometric-thermal variation of machine-tool axes can be obtained as Eq. 6, Eq. 7 and Eq. 8, respectively.

$$
\begin{gathered}
\delta \mathbf{q}_{m}^{C_{k}}(k)=\mathbf{T}_{q i(k)}^{C_{k}} \mathbf{x}_{C_{k}}^{S_{k}} \\
\delta \mathbf{q}_{m}^{S_{k}}(k)=\mathbf{T}_{q i(k)}^{C_{k}} \mathbf{T}_{C_{k}}^{S_{k}} \mathbf{x}_{S_{k}}^{A_{k}^{n}} \\
\delta \mathbf{q}_{m}^{A_{k}^{n}}(k)=\mathbf{T}_{q i(k)}^{C_{k}} \mathbf{T}_{C_{k}}^{S_{k}} \mathbf{T}_{S_{k}}^{A_{k}^{n}} \mathbf{x}_{A_{k}^{n}}^{{ }^{o} F_{k}}
\end{gathered}
$$

The extended EFE model introduces the machininginduced variations into machine tool path error. Therefore, it is feasible to calculate the EFE for machining-induced variations. Then, the limitation of current EFE model can be overcome. The benefits of extending the EFE model and the limitation of current EFE model through a case study will also be discussed in the following section.

\section{Case Study}

A workpiece from [15] will be employed to compare current EFE model and improved EFE model. As show in Fig. 5, the origin of the PCS is $\mathrm{O}_{\mathrm{p}}$. In this case, FCS is taken the same as PCS for sake of simplicity. Only top surface EFGH is milled in this case. The workpiece is located by using the primary datum $\mathrm{ABCD}$, secondary datum $\mathrm{CDHG}$ and tertiary datum BCGF to mill feature $\mathrm{EFGH}$.

The nominal locations of the six locators expressed in ${ }^{0} \mathrm{FCS}$ are shown in Table. 1. The nominal location and orientation of the features in PCS are listed in Table. 2. In the machining, the input errors are intentionally added to the process. Using the above parameters listed in Table. 1 and Table. 2, corresponding EFE models can be established.

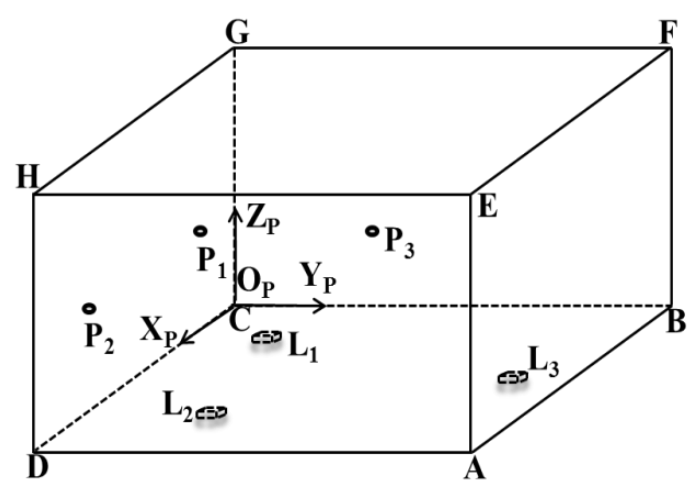

Figure. 5. Workpiece and locating scheme 
Table 1. Nominal position of the locators in ${ }^{0} \mathrm{FCS}$

\begin{tabular}{ccc}
\hline $\begin{array}{c}\text { Locator } \\
\text { index }\end{array}$ & $\begin{array}{c}\text { Datum feature } \\
\text { index }\end{array}$ & $\begin{array}{c}\text { Coordinates of } \\
\text { locators w.r.t }{ }^{0} \text { FCS }\end{array}$ \\
\hline 1 & primary datum ABCD & {$[38,19.1,0]$} \\
2 & primary datum ABCD & {$[19,82.4,0]$} \\
3 & primary datum ABCD & {$[57,82.4,0]$} \\
4 & secondary datum CDHG & {$[19,0,8]$} \\
5 & secondary datum CDHG & {$[57,0,8]$} \\
6 & tertiary datum BCGF & {$[0,50.8,8]$} \\
\hline
\end{tabular}

Table 2. Nominal position and orientation of the features in ${ }^{0} \mathrm{PCS}$

\begin{tabular}{cccc}
\hline $\begin{array}{c}\text { Feature } \\
\text { index } \boldsymbol{i}\end{array}$ & ${ }^{0} \mathbf{w}_{\boldsymbol{i}}$ & $\mathbf{n}_{\boldsymbol{i}}$ & ${ }^{0} \mathbf{t}_{\boldsymbol{i}}$ \\
\hline 1 & {$[\pi, 0,0]$} & {$[0,0,-1]$} & {$[38.1,50.8,0]$} \\
2 & {$[\pi / 2,0,0]$} & {$[0,-1,0]$} & {$[38.1,0,11.43]$} \\
3 & {$[0,-\pi / 2,0]$} & {$[-1,0,0]$} & {$[0,50.8,11.43]$} \\
4 & {$[0,0,0]$} & {$[0,0,1]$} & {$[0,0,20.32]$} \\
\hline
\end{tabular}

Two simple cases are used to discuss the limitation of current EFE model and the benefits of the improved EFE model. In the first case, the machining error without compensation, EFE and compensation value are predicted by current model. In the second case, the machining error without compensation, EFE and compensation value are predicted by the improved model.

In the first case, the datum error machine tool path error and fixture error are intentionally introduced. The datum errors are set as $\mathbf{x}_{d 1}=\left[\begin{array}{llllll}0 & 0 & 0 & 0 & 0 & -0.02\end{array}\right]^{\mathrm{T}}$, $\mathbf{x}_{d 2}=\left[\begin{array}{llllll}0 & 0 & 0 & 0 & 0 & 0\end{array}\right]^{\mathrm{T}}$ and $\mathbf{x}_{d 3}=\left[\begin{array}{llllll}0 & 0 & 0 & 0 & 0 & 0\end{array}\right]^{\mathrm{T}}$. The input to the model that corresponds to the fixture locator error is $\Delta \mathbf{f}(k)=\left[\begin{array}{llllll}0.01 & 0.01 & 0.01 & 0 & 0 & 0\end{array}\right]^{\mathrm{T}}$. The machine path tool error in this case study includes only two parts: the cutting-tool wear induced error $\delta \mathbf{q}_{m}^{q i}=\left[\begin{array}{lllllll}0 & 0 & -0.015 & 0 & 0 & 0\end{array}\right]^{\mathrm{T}}$, and the geometricthermal variation of machine-tool axes induced error $\delta \mathbf{q}_{m}^{A^{n}}=\left[\begin{array}{llllll}0 & 0 & -0.015 & 0 & 0 & 0\end{array}\right]^{\mathrm{T}}$, respectively. Since the machining-induced errors have not been explicitly calculated by current EFE model in [2], only datum error, machine tool path error due to geometric-thermal variation of machine-tool axes and fixture error can be predicted by current EFE model. The machined error of feature $\mathrm{EFGH}$ is $\mathbf{x}_{E F G H}^{P}=\left[\begin{array}{llllll}0 & 0 & 0 & 0 & 0 & -0.045\end{array}\right]^{\mathrm{T}}$. The EFE of machine tool path error calculated by current EFE model is $\Delta \mathbf{m}_{c}=\left[\begin{array}{llllll}0.015 & 0.015 & 0.015 & 0 & 0 & 0\end{array}\right]^{\mathrm{T}}$. The EFE of datum error calculated by Eq. 3 is $\Delta \mathbf{d}=\left[\begin{array}{llllll}0.02 & 0.02 & 0.02 & 0 & 0 & 0\end{array}\right]^{\mathrm{T}} \quad$. By knowing $\Delta \mathbf{d}$, $\Delta \mathbf{m}$ and $\Delta \mathbf{f}$, the total EFE $\Delta \mathbf{t}$ can be calculated out as $\Delta \mathbf{t}=\left[\begin{array}{llllll}0.045 & 0.045 & 0.045 & 0 & 0 & 0\end{array}\right]^{\mathrm{T}}$. Then, the total compensation value for the above errors can be obtained as $\Delta \mathbf{c}=\left[\begin{array}{llllll}-0.045 & -0.045 & -0.045 & 0 & 0 & 0\end{array}\right]^{\mathrm{T}} . \quad$ After adjusting fixture locators under the compensation value $\Delta \mathbf{c}$, the feature EFGH will be milled. With error compensation by current EFE model, the total EFE be cancelled out is $\Delta \mathbf{t}=\left[\begin{array}{llllll}0.045 & 0.045 & 0.045 & 0 & 0 & 0\end{array}\right]^{\mathrm{T}}$, leaving the cutting-tool wear unaddressed.

In the second case, the inputs to the model with compensation that corresponds to the machine tool path error and datum error are set as the same as the previous case. Without error compensation, the machining error of feature EFGH predicted by the improved EFE model is $\mathbf{x}_{E F G H}^{P}=\left[\begin{array}{llllll}0 & 0 & 0 & 0 & 0 & -0.06\end{array}\right]^{\mathrm{T}}$. The EFE of machine tool path error calculated by Eq. 2 and Eq. 8 is $\Delta \mathbf{m}_{i}=\left[\begin{array}{llllll}0.03 & 0.03 & 0.03 & 0 & 0 & 0\end{array}\right]^{\mathrm{T}}$. The EFE of datum error $\Delta \mathbf{d}=\left[\begin{array}{llllll}0.02 & 0.02 & 0.02 & 0 & 0 & 0\end{array}\right]^{\mathrm{T}}$ can be calculated out by Eq. 3. The fixture error is $\Delta \mathbf{f}=\left[\begin{array}{llllll}0.01 & 0.01 & 0.01 & 0 & 0 & 0\end{array}\right]^{\mathrm{T}}$. By knowing $\Delta \mathbf{d}$, $\Delta \mathbf{m}$ and $\Delta \mathbf{f}$, the total EFE $\Delta \mathbf{t}$ can be calculated out as $\Delta \mathbf{t}=\left[\begin{array}{llllll}0.06 & 0.06 & 0.06 & 0 & 0 & 0\end{array}\right]^{\mathrm{T}}$. Then, the total compensation value $\Delta \mathbf{c}=\left[\begin{array}{llllll}-0.06 & -0.06 & -0.06 & 0 & 0 & 0\end{array}\right]^{\mathrm{T}}$ can be obtained. After adjusting fixture locators under the compensation value $\Delta \mathbf{c}$, feature EFGH will be milled. With error compensation by the improved EFE model, the total EFE be cancelled out will be $\Delta \mathbf{t}=\left[\begin{array}{llllll}0.06 & 0.06 & 0.06 & 0 & 0 & 0\end{array}\right]^{\mathrm{T}}$.

\section{Comparison and discussion}

By comparing the results of the current and improved method, it is observed that the improved model can capture more error sources. The machining error of feature EFGH predicted by current EFE model is $\mathbf{x}_{E F G H}^{P}=\left[\begin{array}{llllll}0 & 0 & 0 & 0 & 0 & -0.045\end{array}\right]^{\mathrm{T}}$. This machining error predicted through current model is only caused by datum error, fixture error and the geometric-thermal variation of machine-tool axes induced error, leaving the cutting-tool wear induced error unaddressed. Similarly, only datum error, fixture error and the geometric-thermal variation of machine-tool axes induced error can be equivalent to their corresponding EFEs and compensated by current model. Therefore, the total EFE for the above error sources is $\Delta \mathbf{t}=\left[\begin{array}{llllll}0.045 & 0.045 & 0.045 & 0 & 0 & 0\end{array}\right]^{\mathrm{T}}$.

However, the machining error predicted by improved EFE model is $\mathbf{x}_{E F G H}^{P}=\left[\begin{array}{llllll}0 & 0 & 0 & 0 & 0 & -0.06\end{array}\right]^{\mathrm{T}}$. The difference of the machining errors between current and improved model is understandable because current model can handle more error sources than current model. In addition, the improved EFE model can also cancel out more errors. The total EFE for the above error sources is $\Delta \mathbf{t}=\left[\begin{array}{llllll}0.06 & 0.06 & 0.06 & 0 & 0 & 0\end{array}\right]^{\mathrm{T}}$.

The total compensation values for current model is $\left[\begin{array}{llllll}-0.045 & -0.045 & -0.045 & 0 & 0 & 0\end{array}\right]^{\mathrm{T}}$ and for improved model is $\left[\begin{array}{llllll}-0.06 & -0.06 & -0.06 & 0 & 0 & 0\end{array}\right]^{\mathrm{T}}$. As also can be seen from the above two cases, after adjusting fixture locators 
under their corresponding compensation values for the current and improved models, the improved EFE model can cancel out more error sources than current EFE model. Therefore, after compensating errors by the improved model, machining quality can be improved obviously.

\section{Conclusions}

Current EFE compensation technique did not consider the machining-induced variations. Without compensating these machining-induced variations, the EFE based error compensation methodology will be limited. In order to overcome this limitation, this paper extends current EFE model that includes the machining-induced variations. A case study shows the benefits of the extended EFE model. The improved EFE model can compensate more errors than current EFE model. Compensating more errors by the improved model means that machining quality can be improved obviously.

Its open structure provides a quantitative framework for further expansion. In the future work, the EFE procedure based on DMVs can be applied to investigate error compensation methodology for a general 3-2-1 fixture setup rather than orthogonal 3-2-1 fixture layout. In addition, the EFE model can also expand to deformed workpiece. All of these potential works using EFE model based on DMVs will be pursued and reported in the future.

\section{Acknowledgments}

This work has been partially supported by the National Key Science and Technology Research Program of China (Grant No. 2014ZX04002011-3) and National Natural Science Foundation of China (Grant Nos. 51121063, 51175340, 51775346). The authors would like to thank these financial supports.

\section{References}

[1] Zhou S, Huang Q, Shi J. State space modeling of dimensional variation propagation in multistage machining process using differential motion vectors. IEEE Transactions on Robotics and Automation. 2003;19:296-309.

[2] Du S, Huang D, Lv J. Recognition of concurrent control chart patterns using wavelet transform decomposition and multiclass support vector machines. Computers \& Industrial Engineering. 2013;66:683-695.

[3] Du S, Lv J. Minimal Euclidean distance chart based on support vector regression for monitoring mean shifts of auto-correlated processes. International Journal of Production Economics. 2013;141:377-387.

[4] Du S, Lv J, Xi L. On-line classifying process mean shifts in multivariate control charts based on multiclass support vector machines. International Journal of Production Research. 2012;50:6288-6310.

[5] Tsung F, Li Y, Jin M. Statistical process control for multistage manufacturing and ervice operations: A review and some extensions. International Journal of Services perations and Informatics. 2008; 3 (2), 191-204.

[6] Jin J, Shi J. State space modeling of sheet metal assembly for dimensional control. Journal of Manufacturing Science and Engineering-Transactions of the ASME. 1999;121:756-762.

[7] Huang Q, Shi J, Yuan J. Part dimensional error and its propagation modeling in multi-operational machining processes. Journal of Manufacturing Science and Engineering-Transactions of the ASME. 2003;125:255262.

[8] Djurdjanovic D, Ni J. Dimensional errors of fixtures, locating and measurement datum features in the stream of variation modeling in machining. Journal of Manufacturing Science and Engineering-Transactions of the ASME. 2003; 125:716-30.

[9] Abellan-Nebot JV, Liu J, Romero F. Limitations of the current state space modeling approach in multistage machining processes due to operation variations. 3rd Manufacturing Engineering Society International Conference, Alcoy, Spain. 2009.

[10] Abellan-Nebot JV, Liu J, Subiron FR, Shi J. State space modeling of variation propagation in multistation machining processes considering machining-induced variations. Journal of Manufacturing Science and Engineering -Transactions of the ASME. 2012;134: 1-13

[11] Liu J. Variation reduction for multistage manufacturing processes: A comparison survey of statistical-process-control vs stream-of-variation methodologies. Quality and Reliability Engineering International. 2010; 26: 645-661

[12] Shi J, Zhou S. Quality control and improvement for multistage systems: A survey. IIE Transactions. 2009; 41: 744-753

[13] Abellan-Nebot JV, Subirón FR, Mira JS. Manufacturing variation models in multi-station machining systems. International Journal of Advanced Manufacturing Technology. 2013; 64:63-83

[14] Yang F, Jin S, Li Z. A comprehensive study of linear variation propagation modeling methods for multistage machining processes. International Journal of Advanced Manufacturing Technology. 2017; 90(5): 2139-2151.

[15] Wang H, Huang Q, Katz R. Multi-operational machining processes modeling for sequential root cause identification and measurement reduction. Journal of Manufacturing Science \& Engineering. 2005; 127:512521

[16] Wang H, Huang Q. Error cancellation modeling and its application to machining process control. IIE Transactions. 2006; 38(4): 355-364 